Chem. Ber. 119, 3045- 3058 (1986)

\title{
Tricyclo[5.1.0.0,8]oct-3-en, -oct-4-en und -octan: Darstellung und Thermolyse der Hydroderivate des Octavalens
}

\author{
Manfred Christl*, Clemens Herzog ${ }^{1)}$ und Petra Kemmer \\ Institut für Organische Chemie der Universität Würzburg, \\ Am Hubland, D-8700 Würzburg
}

Eingegangen am 2. Mai 1986

2,3,7,8-Tetrabrombicyclo[4.1.1]oct-3-en (7) ging mit $\mathrm{LiAlH}_{4}$ unter Allylumlagerung in das Tribromid 8 über, das bei der Einwirkung von n-Butyllithium 4-Bromtricyclo[5.1.0.0.8.8 $]$ oct3-en (9) lieferte. Letzteres ergab mit Natrium/tert-Butylalkohol Tricyclo[5.1.0.028.8 $]$ oct-3-en (4). Die Hydrierung von $4 \mathrm{zu}$ Tricyclo[5.1.0.0.8. $]$ octan (6) wurde mit Diimin ausgeführt und war von der Bildung von Bicyclo[5.1.0]oct-2-en (10) begleitet. Mit Natriumiodid brachte 7 das nicht umgelagerte lodid 12 hervor, und die Reduktion von 7 mit Natrium in flüssigem Ammoniak führte in geringer Ausbeute zu Bicycio[4.2.0] octa-3,7-dien (11). $\mathrm{AgNO}_{3}$ wandelte 7 unter Allylumlagerung in das Nitrat 13 um, das mit $\mathrm{LiAlH}_{4}$, wieder unter Allylumlagerung, das Tribromid 14 ergab. Bei der Behandlung von 14 mit n-Butyllithium fiel 4-Bromtricyclo[5.1.0.0 $\left.0^{28}\right]$ oct-4-en (15) an, und daraus wurde mit Natrium/tert-Butylalkohol Tricyclo[5.1.0.0 2.8] oct-4-en (5) gewonnen. Ein neuer Weg zu Octavalen (1), auf dem praktisch kein Cyclooctatetraen als Nebenprodukt entsteht, wurde durch Umsetzung von 4-Bromoctavalen (16) mit tert-Butyllithium und anschließende Hydrolyse gefunden. - Bei $100^{\circ} \mathrm{C}$ ging 4 praktisch quantitativ in Dihydrosemibullvalen (17) über $\left(t_{1 / 2} \approx \mathbf{4 0} \mathrm{min}\right)$. Der Zerfall von 5 lieferte bei $200^{\circ} \mathrm{C}\left(t_{1 / 2} \approx 25 \mathrm{~h}\right)$ ein komplexes Gemisch, in dem nur 11 identifiziert wurde. Der gesättigte Kohlenwasserstoff 6 lagerte bei $160^{\circ} \mathrm{C}$ nahezu quantitativ in ein $5.5: 1-\mathrm{Ge}$ misch aus 3-Methylencyclohepten (18) und 1,3-Cyclooctadien (19) um ( $t_{1 / 2} \approx 8 \mathrm{~h}$ ). Mögliche Mechanismen dieser Thermolysen werden diskutiert.

Tricyclo[5.1.0.02,0]oct-3-ene, -oct-4-ene, and -octane: Preparation and Thermolysis of the Hydro Derivatives of Octavalene

On treatment of 2,3,7,8-tetrabromobicyclo[4.1.1] oct-3-ene (7) with $\mathrm{LiAlH}_{4}$ an allylic rearrangement occurred with formation of tribromide 8, which was converted into 4bromotricyclo[5.1.0.0 2,8$]$ oct-3-ene (9) by n-butyllithium. Sodium/tert-butyl alcohol and 9 reacted to give tricyclo[5.1.0.0 $\left.0^{28}\right]$ oct-3-ene (4). Hydrogenation of 4 was achieved with diimine and afforded tricyclo[5.1.0.0 2,8] octane (6) along with some bicyclo[5.1.0]oct-2-ene (10). Sodium iodide transformed 7 into the unrearranged iodide 12. Reduction of 7 with sodium in liquid ammonia led to bicyclo[4.2.0] octa-3,7-diene (11) in low yield. An allylic rearrangement took place when 7 was treated with $\mathrm{AgNO}_{3}$ to give nitrate 13. In another allylic rearrangement tribromide 14 was formed from 13 and $\mathrm{LiAlH}_{4}$. The reaction of 14 with n-butyllithium afforded 4-bromotricyclo[5.1.0.0.8. (5) was obtained by means of sodium/tert-butyl alcohol. By treatment of 4-bromooctavalene (16) with tert-butyllithium and subsequent hydrolysis, a new route to octavalene (1) was elaborated with virtually no formation of cyclooctatetraene as side product. - At $100^{\circ} \mathrm{C} 4$ rearranged $\left(t_{1 / 2} \approx 40 \mathrm{~min}\right.$ ) almost quantitatively to dihydrosemibullvalene (17). The decomposition of 5 at $200^{\circ} \mathrm{C}\left(t_{12} \approx 25 \mathrm{~h}\right)$ gave a complex mixture. in which only 11 has been

(C) VCH Verlagsgesellschaft mbH, D-6940 Weinheim, 1986

$0009-2940 / 86 / 1010-3045 \$ 02.50 / 0$ 
identified. The saturated hydrocarbon 6 was converted virtually quantitatively into a 5.5:1 mixture of 3-methylenecycloheptene (18) and 1,3 -cyclooctadiene $(19)$ at $160^{\circ} \mathrm{C}\left(t_{1 / 2} \approx 8 \mathrm{~h}\right)$. Possible mechanisms of these rearrangements are discussed.

Die Eigenschaften von Bicyclo[1.1.0]butan-Derivaten hängen vom Winkel zwischen ihren beiden Dreiringen $a b^{2-5)}$, der in der Stammsubstanz $121.7^{\circ}$ miBt ${ }^{6}$ und durch Substituenten beeinflußt wird ${ }^{\text {s) }}$. Zur Einstellung besonders kleiner Interplanarwinkel bewährte sich die Uberbrückung der endo-2,4-Positionen. Eine direkte Bindung führt zum minimalen Wert von $70.5^{\circ}$ in Tetra-tert-butyltetrahedran ", die Einkohlenstoflbrücke in Tricyclo-

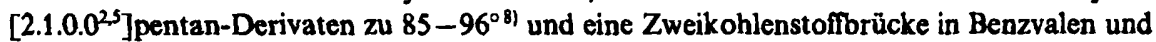
anderen Tricyclo[3.1.0.0 $0^{2,6}$ hexan-Abkömmlingen $\mathrm{zu} 106^{\circ 9}$ bzw. ca. $112^{\circ 8 \mathrm{~b}, 10}$. Dagegen hat eine Brücke aus zwei C-Atomen und einem S-Atom in 5-Thiabenzohomobenzvalen einen Interplanarwinkel von $122^{\circ 11}$ zur Folge, der praktisch mit dem des Bicyclobutans ${ }^{(\mathfrak{)}}$ übereinstimmt.

Durch die Synthese von Octavalen (1) ${ }^{12)}$ hatten wir erstmals ein Bicyclobutan-Derivat mit einer Vierkohlenstofibrücke erhalten. Modellbetrachtungen zeigen, daß sich in $1 \sigma-$ und $\pi$-Elektronen-System gegenseitig aufweiten sollten ${ }^{126)}$ und so ein besonders großer Bicyclobutan-Interplanarwinkel vorliegen könnte. MNDO-Rechnungen ergaben allerdings für 1 nur einen Wert von $124^{\circ}{ }^{(3)}$ und weisen damit auf eine Resistenz gegen die WinkelvergröBerung hin, obwohl bei 1,3-Diphenylbicyclo[1.1.0]butan-endo-2,endo-4-dicarbonsäure-dimethylester das bisherige Maximum von $127^{\circ}$ gefunden worden war ${ }^{5)}$. Im Diels-Alder-Addukt 2 von 1 überspannt ebenfalls eine Kette aus vier C-Atomen das $\sigma$-Elektronen-System, jedoch ergibt sich wegen der $z_{\text {wei }} \mathrm{sp}^{3}$-hybridisierten Zentren ein nahezu normaler Interplanarwinkel von $122.7^{14}$.

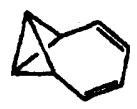

1

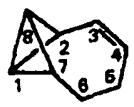

4

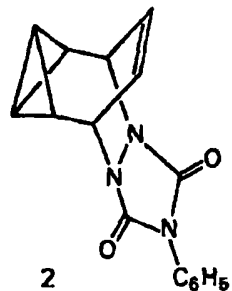<smiles>COc1ccc2c(c1)OC1CC1C1CC21</smiles>

3

Uber 1 und drei seiner Derivate ${ }^{12 b)}$ sowie 2 hinaus ist kürzlich als weiteres mit einer Kette aus vier Atomen überbrücktes Bicyclobutan der Heterocyclus 3 auf photochemischem Weg bereitet worden ${ }^{15)}$. Um diesen Einzelbefunden eine systematische Untersuchung an die Seite zu stellen, synthetisierten wir jetzt die Titelverbindungen 4-6. Insbesondere sollten die Thermolysen, die Photoelektronenspektren ${ }^{(3)}$ und die ${ }^{13} \mathrm{C}$-NMR-Spektren ${ }^{16)}$ studiert bzw. analysiert werden. Die ${ }^{13} \mathrm{C}$-NMR-chemischen Verschiebungen der Bicyclobutan-Brückenkopf-C-Atome von $1\left(\delta=-13.4^{(2)}\right)$ einerseits und $2\left(11.5,20.2^{14}\right)$ sowie $3\left(15.6^{15}\right)$ andererseits differieren so stark, daß eine Anomalie zu vermuten war, über die wir mit Hilfe der Werte von 4-6 mehr zu erfahren hofften.

Chem. Ber. 119 (1986) 


\section{A. Synthesen}

Als Ausgangssubstanz bot sich das aus Benzvalen über Homobenzvalen gut zugängliche Tetrabromid 7 an, das als Vorstufe für 4-Bromoctavalen (16) gedient hatte ${ }^{12 b)}$. Die Reaktion mit Lithiumaluminiumbydrid verlief unter Allylumlagerung und erbrachte das Tribromid $8 \mathrm{mit} 70 \%$ Ausbeute. In Analogie zur Synthese von $1^{12)}$ führte die Behandlung von 8 mit n-Butyllithium zur Knüpfung der zentralen Bicyclobutan-Bindung durch Eliminierung der Bromatome am Vierring, woraus 4-Bromtricyclo[5.1.0.0 28 ] oct-3-en (9) mit 69\% Ausbeute hervorging. Reduktion mit Natrium/tert-Butylalkohol wandelte 9 in den Zielkohlenwasserstoff 4 um $(60 \%)$. Die Einwirkung von Diimin, das sich schon zur Hydrierung der Doppelbindung von Benzvalen ${ }^{17 \mathrm{a})}$ und 1-Methylbenzvalen ${ }^{17 \mathrm{~b})}$ bewährt hatte, auf 4 lieferte die gesättigte Stammverbindung 6(52\%), wobei wir uns zur Erzeugung von Diimin der Methode von Reese et al. ${ }^{18)}$ bedienten. Allerdings trat dabei Bicyclo[5.1.0]oct2-en (10) als Nebenprodukt auf $(6: 10=8: 1)$, das wohl aus 6 unter der katalytischen Einwirkung von Protonenspuren entstanden war. Die Empfindlichkeit gegenüber Säuren ist typisch für die Bicyclobutane. Insbesondere unterliegen Tricyclo $\left[4.1 .0 .0^{2.7}\right]$ heptan $^{19 a)}$ und Tricyclo[3.1.0.0 $\left.0^{2.6}\right]$ hexan $^{19 b)}$, die niedrigen Homologen von 6, der gleichen Isomerisierung.

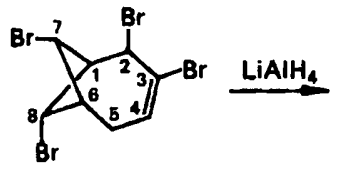

7<smiles>BrC1=CC2C(Br)C(C1)C2Br</smiles>

$8(70 \%)$

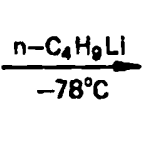<smiles>BrC12CCC3C4C5C3C1C5C42</smiles>

9 (69\%)
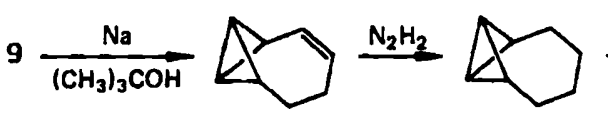

$6(52 \%)$

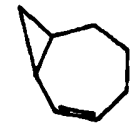

10

Für die Bereitung des symmetrischen Dihydrooctavalens (5) ausgehend von 7 war es erforderlich, die Position der Doppelbindung zu erhalten. Der denkbar kürzeste Weg, nämlich der Ersatz der Bromatome am Allyl-System durch HAtome und die Wurtz-Reaktion zur Bildung der Bicyclobutan-Zentralbindung in einem Arbeitsgang durch Natrium in flüssigém Ammoniak, führte nicht zum Ziel 5, sondern mit geringer Ausbeute zu dessen Valenzsisomerem 11. Im nächsten Plan sahen wir vor, das allylständige Bromatom von 7 in einer $S_{N} 2^{\prime}$-Reaktion durch ein Iodid-Ion $z u$ verdrängen, um nach einem erneuten $S_{N} 2^{\prime}$-Prozeß mit $\mathrm{LiAlH}_{4}$ zum Tribromid $14 \mathrm{zu}$ gelangen. Auch dies schlug fehl, weil der erste Schritt formal als $S_{N}$ 2-Substitution ablief und das entstandene 12 bei der Reduktion wie erwartet in 8 überging. Weil eine $S_{N} 2-$ Reaktion an C-2 von 7 unter extremer sterischer Hinderung leiden dürfte, vermuten wir für die Umwandlung $7 \rightarrow 12$ zwei aufeinanderfolgende $S_{\mathrm{N}} 2^{\prime}$-Schritte im Sinne der Einstellung eines Gleichgewichts ${ }^{20}$, in dem 12 die Oberhand hat. Um dies zu überprüfen, wäre die

Chem. Ber. 119 (1986) 
Kenntnis der Konfiguration von C-2 in 7 und 12 erforderlich. Aus den NMRSpektren läßt sich diese Information aber nicht gewinnen.

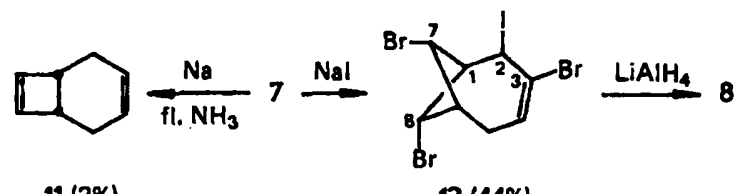

$12(44 \%)$

Im dritten Anlauf wollten wir 7 unter Allylumlagerung in den zugehörigen Alkohol überführen, um nach Umwandlung der Hydroxylfunktion in eine Abgangsgruppe diese wieder in cinem $\mathrm{S}_{\mathrm{N}} 2^{\prime}$-Schritt durch ein Hydrid-Ion zu ersetzen, woraus 14 hervorgehen müßte. Uberraschenderweise gestaltete sich die Verwirklichung die Plans ökonomischer.

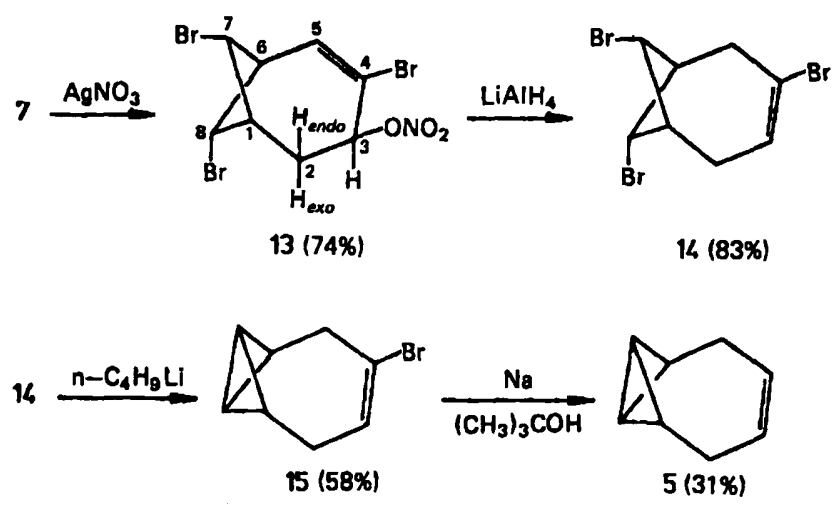

Die Reaktion von 7 mit Silbernitrat in wäBrigem Aceton erbrachte nämlich nicht den erhofften Alkohol, sondern dessen Nitrat 13, das dann in absolutem Aceton mit $74 \%$ Ausbeute erhalten wurde. Die Bildung von Nitraten aus Halogeniden unter diesen Bedingungen ist bekannt, und auch ein Fall mit Allylumlagerung wird erwähnt ${ }^{21}$. Unerwartet folgte die Reduktion von 13 mit $\mathrm{LiAlH}_{4}$ nicht der normalen Route, wonach unter Spaltung der NO-Bindung der zugehörige Alkohol entsteht ${ }^{22)}$. Statt dessen diente das Nitrat-Ion als Abgangsgruppe, aber nicht im Sinne der bekannten direkten Substitution ${ }^{23)}$, sondern wieder in einem $S_{\mathrm{N}} 2^{\prime}$-Prozeß, so daß die ursprünglich beabsichtigte zweistufige Transformation $7 \rightarrow 14$ doch erreicht werden konnte. Zum Zielkohlenwasserstoff 5 gelangten wir dann durch Anwendung des bei der Transformation $8 \rightarrow 4$ erprobten Verfahrens. - Die Einwirkung von n-Butyllithium auf 14 führte unter Bildung der BicyclobutanEinheit mit 58\% Ausbeute zu 4-Bromtricyclo[5.1.0.0.28] oct-4-en (15), und daraus wurde 5 mit Natrium/tert-Butylalkohol dargestellt (31\% Ausbeute).

Bei unserer Synthese des unsubstituierten Octavalens (1) fallt ein 4:1-Gemisch aus 1 und Cyclooctatetraen an ${ }^{(2)}$, das bisher nicht getrennt werden konnte. Für die Photoelektronenspektroskopie ${ }^{13)}$ war aber möglichst reines 1 wünschenswert. Es zeigte sich jetzt, daß das in reiner Form darstellbare 4-Bromoctavalen (16) ${ }^{12 b}$ 
durch Brom-Lithium-Austausch mit tert-Butyllithium und anschließende Hydrolyse - dieses Verfahren hatten wir schon zur Bereitung einiger in 4-Stellung unsubstituierter Homobenzvalene angewandt ${ }^{24}$ - in 1 umgewandelt werden kann, das so gut wie kein Cyclooctatetraen enthält.

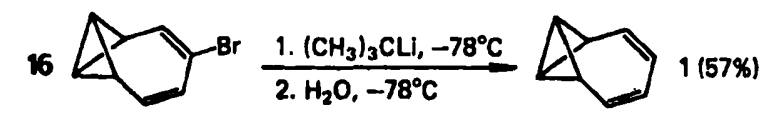

Die NMR-Spektren aller Verbindungen bieten zuverlässige Belege für die Struktur. In den Bicyclo[4.1.1] ]octan-Derivaten 8 und 12-14 ist im Vergleich zum Ausgangsmaterial 7 die Konfiguration von C-7,8 nicht verändert. Dies manifestiert sich in der Multiplizität der 7,8-H-Signale. Die Lage der Doppelbindung leitet sich vom Aufspaltungsmuster der Absorptionen des Vinylprotons und der Brückenkopfprotonen sowie von der Größe der zugehörigen Kopplungskonstanten ab. So ist $J_{1,2}=9.5 \mathrm{~Hz}$ in 8 und $J_{5,6}=9.8 \mathrm{~Hz}$ in 13 charakteristisch groB für die vicinale Beziehung zwischen Vinyl- und Brückenkopfproton, während in 12 und 14 Wechselwirkungen von $<1 \mathrm{~Hz}$ bzw. $1.0 \mathrm{~Hz}$ mindestens vier Bindungen zwischen diesen Kernen anzeigen.

Die Konfiguration von $\mathbf{C}-2$ in 12 und $\mathrm{C}-3$ in 13 geht wie jene von $\mathrm{C}-2$ in $7^{120)}$ nicht aus den NMR-Spektren hervor, da 7,8-H nicht mit den Protonen der Vier-Kohlenstoff-Brücke koppeln. Allerdings liegt die räumlich günstigere Anordnung des Halogenatoms an C-2 cis zu C-7 nahe, weil $7^{12 b}$ und 12 (siehe oben) wahrscheinlich unter thermodynamischer Steuerung entstehen. Bei $\mathrm{S}_{\mathrm{N}} \mathbf{2}^{\prime}-\mathrm{Reaktionen}$ ist sowohl der cis- als auch der trans-Angriff des $\mathrm{Nu}$ cleophils relativ zur Abgangsgruppe möglich ${ }^{25}$ ). Deshalb nehmen wir für die Nitratfunktion in 13 ebenfalls die sterisch bevorzugte cis-Stellung relativ zu C-7 (endo-Stellung) an. Unter dieser Voraussetzung haben wir das ${ }^{1} \mathrm{H}$-NMR-Spektrum interpretiert, das extrem unter-

Tab. 1. ${ }^{1} \mathrm{H}$-NMR-Chemische Verschiebungen ( $\delta$-Werte) und Kopplungskonstanten (in $\mathrm{Hz}$ ) von Tricyclo[5.1.0.0.2. $]$ octan-Derivaten in $\mathrm{CDCl}_{3}$

\begin{tabular}{|c|c|c|c|c|c|c|c|c|c|}
\hline Nr. & $1,8-H$ & $2-\mathrm{H}$ & 3-H & $4-\mathrm{H}$ & $5-\mathrm{H}$ & $6-\mathrm{H}$ & $7-\mathrm{H}$ & $J_{1,2}{ }^{a)}$ & $J_{2,7}$ \\
\hline $1^{b)}$ & 1.27 & 2.97 & 6.13 & 5.72 & 5.72 & 6.13 & 2.97 & 3.0 & c) \\
\hline $4^{d)}$ & $\begin{array}{l}t \\
1.79 \\
t\end{array}$ & $\begin{array}{l}\mathrm{m} \\
2.73 \\
\mathrm{dqd}\end{array}$ & $\begin{array}{l}\mathrm{m} \\
5.73 \\
\mathrm{ddt}\end{array}$ & $\begin{array}{l}\mathrm{m} \\
5.58 \\
\mathrm{dtd}\end{array}$ & $\begin{array}{l}\mathrm{m} \\
2.13 \\
\mathrm{~m}\end{array}$ & $\begin{array}{l}\mathbf{m} \\
1.80 \\
\mathbf{m}\end{array}$ & $\begin{array}{r}\mathrm{m}_{2.85} \\
\approx \operatorname{sext}\end{array}$ & 3.4 & 3.4 \\
\hline 5 & $\begin{array}{l}1.54 \\
t^{(0)}\end{array}$ & $\begin{array}{l}2.85 \\
\mathrm{~m}\end{array}$ & $\begin{array}{l}2.56 \\
\mathrm{~m}\end{array}$ & $\begin{array}{l}5.35 \\
\mathrm{~m}\end{array}$ & $\begin{array}{l}5.35 \\
\mathrm{~m}\end{array}$ & $\begin{array}{l}2.56 \\
\mathrm{~m}\end{array}$ & 2.85 & 3.8 & c) \\
\hline 6 & $\begin{array}{l}1.23 \\
t\end{array}$ & $\begin{array}{l}2.83 \\
\mathrm{~m}\end{array}$ & $\begin{array}{l}1.72 \\
\mathrm{~m}\end{array}$ & $\begin{array}{l}1.32 \\
\mathrm{~m}\end{array}$ & $\begin{array}{l}1.32 \\
\mathrm{~m}\end{array}$ & $\begin{array}{l}1.72 \\
\mathrm{~m}\end{array}$ & $\begin{array}{l}2.83 \\
\mathrm{~m}\end{array}$ & 3.8 & c) \\
\hline $9^{n}$ & $\begin{array}{l}1.85 \\
t\end{array}$ & $\underset{\mathrm{m}}{\approx 2.66}$ & $\begin{array}{l}6.10 \\
d\end{array}$ & - & $\underset{\mathrm{m}}{\approx 2.66}$ & $\begin{array}{l}1.82 \\
\mathrm{~m}\end{array}$ & $\begin{array}{r}2.85 \\
\approx \operatorname{sext}\end{array}$ & 3.0 & $\approx 3.4$ \\
\hline $15^{2}$ & $\begin{array}{l}1.59 \\
t^{e)}\end{array}$ & $\begin{array}{r}2.70 \\
\approx \text { sext }\end{array}$ & $\begin{array}{l}3.03 \\
\mathrm{~m}\end{array}$ & - & $\begin{array}{l}5.84 \\
\text { tq }\end{array}$ & $\begin{array}{l}2.43 \\
\mathrm{~m}\end{array}$ & $\begin{array}{c}2.81 \\
\approx \text { sextd }\end{array}$ & 3.7 & 3.4 \\
\hline
\end{tabular}

a) $J_{12}=J_{1,7}=J_{2,8}=J_{7,8}-{ }^{\text {b) }}$ Chemische Verschiebungen aus Lit. ${ }^{12)}$. Durch Entkoppeln des $2,7-\mathrm{H}^{-S i g n a l s}$ war es möglich, das $\mathrm{AA}^{\prime} \mathrm{XX}^{\prime}$-Spektrum ${ }^{26)}$ von $3,4,5,6-\mathrm{H}$ zu analysieren: $J_{3,4}=+11.4, J_{3,5}=+1.3, J_{3,6}=+0.9, J_{4,5}=+6.7 \mathrm{~Hz}-{ }^{\text {c) }}$ Nicht ermittelt. - d $J_{2,3}=$ $5.6, J_{24}=0.6, J_{3,4}=11.0, J_{3,5}=1.2, J_{4,4}=5.8, J_{6,7}=4.5 \mathrm{~Hz}-{ }^{\circ}$ ) Weitere Aufspaltung (pseudo-quint) mit Linienabstand $0.7 \mathrm{~Hz}$ durch Kopplung mit $3-\mathrm{H}_{2}$ und $6-\mathrm{H}_{2},-\mathrm{J}_{2,3}=$ $6.3, J_{6,7} \approx 4.5 \mathrm{~Hz} .-8 J_{2,3}=J_{6,7}=3.1, J_{3,5}=J_{5,7}=1.5, J_{5,6}=4.5 \mathrm{~Hz}$.

Chem. Ber. 119 (1986) 
schiedliche Kopplungskonstanten der beiden $2-\mathrm{H}$ zu 1-H $(7.5,1.5 \mathrm{~Hz})$ und 3-H $(1.9,6.0 \mathrm{~Hz})$ aufweist und damit ein Abweichen von $C-2$ und $C-3$ aus der durch $C-1,4,5,6$ definierten Ebene nach der endo- bzw. exo-Seite nahelegt. Diese Konformation dürfte ihre Stabilität von der günstigen pseudoäquatorialen Stellung der Nitratgruppe und der gestaffelten Anordnung aller Substituenten an C-2,3 beziehen.

Soweit sie spezifiziert sind, beruhen die Zuordnungen in den ${ }^{13} \mathrm{C}-\mathrm{NMR}$-Spektren von 8 und 12-14 auf dem Vergleich mit den Daten verwandter Verbindungen wie $7^{12 b}$, auf den üblichen Substituenteneffekten und, zur Identifizierung der Signale von C-1,6, auf der Größe von Restkopplungen in den off-resonance-entkoppelten Spektren, wo eine Messung unter Protonenkopplung nicht vorgenommen wurde.

Das Bicyclobutan-System in 4-6, 9 und 15 verursacht in den ${ }^{2} \mathrm{H}-\mathrm{NMR}$-Spektren, deren Daten in Tab. 1 gesammelt sind, das charakteristische Triplett von $1,8-\mathrm{H}$ bei $\delta=1.23-1.85$ mit $J_{1,2}=J_{1,7}=J_{2,8}=J_{7,8}=3.0-3.8 \mathrm{~Hz}$. DaB dieses Signal bei Octavalen (1) trotz der beiden CC-Doppelbindungen in der Nähe der Absorption des gesättigten 6 liegt und gegenüber dem Triplett des Monoolefins 4 um $0.52 \mathrm{ppm}$ zu höherem Feld verschoben ist, spricht für einen speziellen Hochfeldeffekt in 1, der in den ${ }^{13}$ C-NMR-Spektren deutlicher zu Tage tritt ${ }^{16}$. Hier sei nur auf die für das Bicyclobutan-System typischen Parameter, nämlich die Lage der C-1,8-Absorption $(\delta=10.51-12.94$ ) und ihre Kopplungskonstanten über eine Bindung (190-200 Hz) hingewiesen. Die Zuordnung der Signale zu C-5 und C-6 in 416) und 9 wurde aufgrund des Substituenteneffekts des Bromatoms in 9 und der Feinstrukturen in den protonengekoppelten Spektren vorgenommen.

\section{B. Thermolysen}

Die neuen Kohlenwasserstoffe 4-6 wurden unter sorgfältigem Ausschlu $B$ von Säurespuren in trockenem $\left[D_{6}\right]$ Benzol thermolysiert. Schon bei $100^{\circ} \mathrm{C}$ bildete sich aus $\mathbf{4}$ mit einer Halbwertszeit von ca. $\mathbf{4 0}$ min mit nahezu quantitativer Ausbeute Dihydrosemibullvalen (17), das anhand seines ${ }^{1}$ H-NMR-Spektrums durch Vergleich mit Literaturangaben ${ }^{27}$ identifiziert wurde. Das Spektrum bei $400 \mathrm{MHz}$ ließ sich vollständig analysieren (Tab. 2). Als wesentlich weniger labil erwies sich das gesättigte 6. Es lieferte bei $160^{\circ} \mathrm{C}$ mit einer Halbwertszeit von ca. 8 h ebenfalls mit nahezu quantitativer Ausbeute ein 5.5:1-Gemisch aus 3-Methylencyclohepten (18) und 1,3-Cyclooctadien (19). Die Charakterisierung dieser Produkte gelang

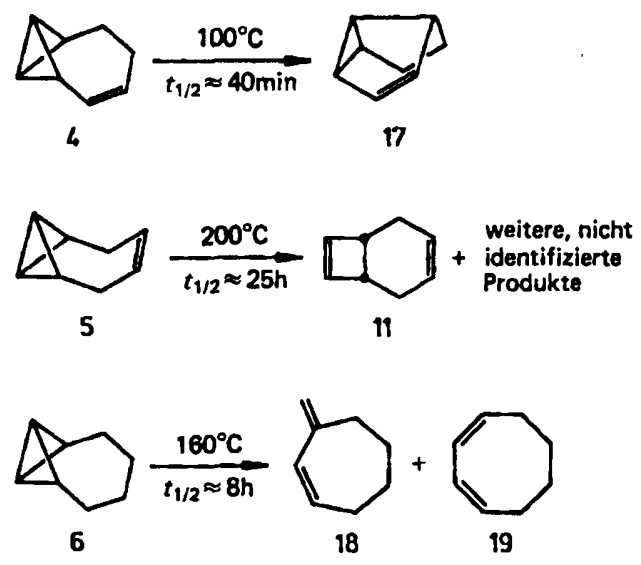

Chem. Ber. 119 (1986) 
anhand der bekannten NMR-Daten $\left(18^{28)}, 19^{29}\right)$. Gegenüber der Hitze als noch resistenter zeigte sich 5 , das erst bei $200^{\circ} \mathrm{C}$ mit einer Halbwertszeit von ca. $25 \mathrm{~h}$ zu einem komplexen Gemisch zerfiel, in dem durch Spektrenvergleich Bicyclo[4.2.0]octa-3,7-dien (11) als eine der wichtigeren Komponenten erkannt wurde.

Die Umlagerung $4 \rightarrow 17$ interpretieren wir als suprafaciale [1,3]-C-Wanderung unter Retention mit dem Diradikal 20 als Zwischenstufe. Somit nimmt die Thermolyse von 4 einen anderen Verlauf als jene des niedrigeren Homologen 21, das sich bei $135^{\circ} \mathrm{C}$ in 23 umwandelt, wobei mit $(E, Z, Z)$-Cycloheptatrien (22) als Zwischenstufe zu rechnen ist ${ }^{196}$. Unter dem Eindruck der Rechnungen von Dewar und Kirschner ${ }^{30)}$ hatten wir für das $\mathrm{zu} 20$ analoge Diradikal als Zwischenstufe beim Ubergang $21 \rightarrow 22$ argumentiert, halten daran aber aufgrund der kinetischen Untersuchung der Thermolyse von 1- und 2-Phenyltricyclo[4.1.0.0 $\left.{ }^{2,7}\right]$ heptan ${ }^{31}$ nicht mehr fest. Bicyclo[4.2.0]octa-2,7-dien, das höhere Homologe von 23, kann als Zwischenstufe beim Zerfall von 4 ausgeschlossen werden, da diese Verbindung unter den Thermolysebedingungen stabil wäre ${ }^{32}$. Diradikalische $\mathrm{Z}$ wischenstufen wurden auch bei der Umlagerung nichtüberbrückter Bicyclobutan-Derivate diskutiert ${ }^{33)}$

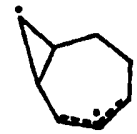

20

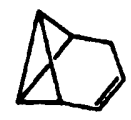

21

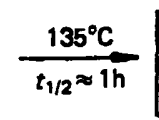

22

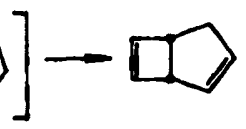

23

Auch 6 lagert nicht in der Weise seines niedrigeren Homologen 24 um, das wohl in einem zweistufigen Prozeß mit 25 als Intermediat Bicyclo[3.2.0]hept-6-en (26) hervorbringt ${ }^{196,34)}$. Das zu 26 analoge Bicyclo[4.2.0]oct-7-en tritt bei der Thermolyse von 6 nicht auf, weil es unter den Reaktionsbedingungen stabil ${ }^{35}$ und damit anband seiner NMR-Spektren identifiziert worden wäre. Als gemeinsame Zwischenstufe für die Produkte 18 und 19 schlagen wir das Carben 27 vor, das durch Insertion des zweibindigen C-Atoms in die benachbarte CH-Bindung in 18 und in eine benachbarte CC-Bindung in 19 übergehen könnte. Ob 27 ein Diradikal des Typs 20 vorgelagert ist, bleibt offen. Umwandlungen von endo,endo-überbrückten Bicyclobutanen in 3-Methylencycloalkene sind in der Reihe der [3.1.1]und [4.1.1] Propellane als thermische Prozesse ${ }^{36)}$ und bei einer Reihe von Derivaten von 24 als Silber-Ionen-katalysierte Reaktionen ${ }^{37}$ bekannt.

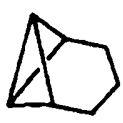

24

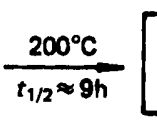

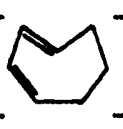

25

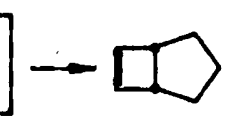

26<smiles>CC1CCCCCC1</smiles>

27

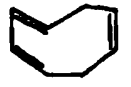

28

Während 4 und 6 thermolabiler sind als ihre niedrigeren Homologen 21 bzw. 24, worin sich möglicherweise die stärkere Spannung des durch eine Kette aus vier C-Atomen überbrückten Bicyclobutan-Systems ausdrückt, übertrifft die Hitzebeständigkeit von 5 sogar jene von 24. Anders als bei 4 ist die Doppelbindung

Chem. Ber. 119 (1986) 
in 5 nicht in Konjugation mit dem gespannten $\sigma$-System und kann so dessen Offnung nicht erleichtern. Die Röntgenstrukturanalyse des Derivats $2^{14)}$ von 5 zeigte keine besondere Aufweitung des Bicyclobutan-Interplanarwinkels. Ein im Vergleich zu 2 ähnliches Abweichen von C-4,5 aus der von C-2,3,6,7 gebildeten Ebene durch Abwinkelung an C-3 und C-6 sollte 5 in die Lage versetzen, die Spannungsenergie, wenigstens gegenüber 4 und 1, zu verkleinern. Dies könnte die Eigenschaften von 5 denen von 24 nahebringen und so den Ubergang in 11 erkJären. Da der direkte Übergang eines endo,endo-überbrückten Bicyclobutans in ein cis-anelliertes Cyclobuten gemäß den Woodward-Hoffmann-Regeln und dem Dewar-Evans-Zimmerman-Prinzip verboten ist, wäre $(E, Z, Z)-1,3,6$-Cyclooctatrien (28) die wahrscheinlichste $Z$ wischenstufe auf dem Weg zu 11. Bedingt durch die hohe Temperatur dürften, wie das Produktgemisch anzeigt, neben der thermisch induzierten Umlagerung von 5 auch andere Reaktionen zum Zuge kommen, etwa durch Säurespuren ausgelöste, die bei der Thermolyse von 24 durch sorgfältige Probevorbereitung unterbunden ${ }^{34 b)}$, bei jener seines 1-Phenylderivats ${ }^{31)}$ aber nicht mehr ganz ausgeschaltet werden konnten.

Auf den Mechanismus der Umlagerung von Octavalen (1), die bei $50^{\circ} \mathrm{C}$ mit einer Halbwertszeit von ca. $5 \mathrm{~h} \mathrm{zu}$ Cyclooctatetraen führt ${ }^{12 b)}$, ergeben sich aus obigen Thermolysen keine zwingenden Hinweise. Beim thermisch ähnlich labilen Benzvalen dürfte Benzol unter Mitwirkung der Vinylenbrücke in einem einstufigen Sechs-Elektronenproze $B$ entstehen ${ }^{38}$. Wegen der Beteiligung von acht Elektronen ist der analoge Vorgang bei 1 verboten. Als Zwischenstufe erscheint das mit 27 verwandte Cycloheptatrienylcarben wenig wahrscheinlich, weil dieses zum Teil auch Heptafulven liefern sollte ${ }^{39}$. Jedoch sind das zu 20 analoge Diradikal und $(E, Z, Z, Z)$-Cyclooctatetraen mögliche Kandidaten, die in einer Cyclopropyl-AllylRadikal-Umlagerung bzw. über Bicyclo[4.2.0]octa-2,4,7-trien ${ }^{40)}$ in Cyclooctatetraen übergehen könnten.

Wir danken der Deutschen Forschungsgemeinschaft und dem Fonds der Chemischen Industrie für finanzielle Förderung sowie der Union Rheinische Braunkohlen Kraftstoff AG, Wesseling, für Spenden von Dimethylether, dem Lösungsmittel bei der Benzvalen-Darstellung.

\section{Experimenteller Teil \\ Geräte: Lit. ${ }^{\text {(2b). }}$}

\section{A. Synthesen}

3,exo-7,syn-8-Tribrombicyclo[4.1.1 Joct-2-en (8): Zur Suspension von $950 \mathrm{mg}$ (25.0 mmol) Lithiumaluminiumhydrid in $100 \mathrm{ml}$ absol. Ether tropfte man bei $20^{\circ} \mathrm{C}$ unter Rühren innerhalb von $30 \mathrm{~min} 9.50 \mathrm{~g}(22.4 \mathrm{mmol}) 7^{12 \mathrm{~b})}$ in $200 \mathrm{ml}$ absol. Ether. Man rührte weitere $15 \mathrm{~h}$ bei $20^{\circ} \mathrm{C}$, kochte dann 3 h unter Rückfluß, gab unter Kühlung mit Eis vorsichtig die gerade notwendige Menge Wasser zu, trennte die Etherphase ab, extrahierte den Hydroxidniederschlag dreimal mit je $100 \mathrm{ml}$ Dichlormethan, trocknete die vereinigten organischen Phasen mit $\mathrm{Na}_{2} \mathrm{SO}_{4}$ engte i. Vak. ein und löste den festen Rückstand aus Methanol um. Einschließlich der aus der Mutterlauge gewonnenen Fraktion ergaben sich $5.40 \mathrm{~g}(70 \%) 8$ als farblose Kristalle mit Schmp. $122-123^{\circ} \mathrm{C}$. - IR (KBr): $1640 \mathrm{~cm}^{-1}(\mathrm{C}=\mathrm{C})$. $-\mathrm{MS}(70 \mathrm{eV}): \mathrm{m} / \mathrm{z}=$ 
$348,346,344,342\left(0.5,1.5,1.6,0.6 \%, \mathrm{M}^{+}\right), 104(100) .-{ }^{1} \mathrm{H}-\mathrm{NMR}\left(\mathrm{CDCl}_{3}\right): \delta=2.04-2.20$ $(\mathrm{m} ; 2 \mathrm{H}), 2.83-3.00(\mathrm{~m} ; 2 \mathrm{H}), 3.11(\mathrm{~m}, 6-\mathrm{H}), 3.29$ (ddd, $J_{1,2}=9.5, J_{1,6}=5.5, J_{1,8}=7.4 \mathrm{~Hz}$; 1-H), $4.40(\mathrm{~s} ; 7-\mathrm{H}), 5.46\left(\mathrm{t}, J_{6,8}=7.4 \mathrm{~Hz} ; 8-\mathrm{H}\right), 6.19(\mathrm{dm} ; 2-\mathrm{H}) .-{ }^{13} \mathrm{C}-\mathrm{NMR}\left(\mathrm{CDCl}_{3}\right): \delta=$ 25.29 (t, $129 \mathrm{~Hz}$; C-5), 37.13 (t, $128 \mathrm{~Hz} ; \mathrm{C}-4$ ), 44.80 (d, $170 \mathrm{~Hz}$ ) und 51.63 (d, $160 \mathrm{~Hz}$ ) (C-7,8, spezifische Zuordnung dieser Signale in Lit. $\left.{ }^{16}\right), 51.44(\mathrm{~d}, 149 \mathrm{~Hz})$ und $52.90(\mathrm{~d}, 150 \mathrm{~Hz})$ (C-1,6), 126.63 (s; C-3), 128.61 (d, $169 \mathrm{~Hz}$; C-2).

$\mathrm{C}_{8} \mathrm{H}_{9} \mathrm{Br}_{3}$ (344.9) Ber. C 27.86 H 2.63 Br 69.51 Gef. C 28.59 H 2.98 Br 69.04

Verbindung 8 entstand auch (59\% Ausb.), als 12 (siehe unten) mit Lithiumaluminiumhydrid gemäß obiger Vorschrift (Ansatzgröße $1.0 \mathrm{mmol}$ ) behandelt wurde.

4-Bromtricyclo[5.1.0.0.8.8 Joct-3-en (9): Unter Stickstoff und Rühren tropfte man innerhalb von $30 \mathrm{~min} 19.8 \mathrm{mmol} \mathrm{n}$-Butyllithium (1.2 $\mathrm{N}$ in Hexan) zur Suspension von $6.20 \mathrm{~g}$ (17.9 mmol) 8 in $100 \mathrm{ml}$ absol. Ether, die auf $-78^{\circ} \mathrm{C}$ gekühlt war. Man ließ während $1 \mathrm{~h}$ auf $0^{\circ} \mathrm{C}$ erwärmen und rührte dann das Reaktionsgemisch noch $40 \mathrm{~min}$ im Eisbad. Nach vorsichtiger Hydrolyse trennte man die Phasen, extrahierte die wäßrige Phase dreimal mit je $25 \mathrm{ml}$ Ether, trocknete die vereinigten organischen $\mathrm{Phasen}$ über $\mathrm{Na}_{2} \mathrm{SO}_{4}$ und engte $\mathrm{i}$. Vak. ein. Aus dem Rückstand destillierten bei $70-71^{\circ} \mathrm{C} / 4-5$ Torr $2.30 \mathrm{~g}(69 \%)$ farbloses, flüssiges 9 . IR (Film): $1652 \mathrm{~cm}^{-1}(\mathrm{C}=\mathrm{C})$. - MS (70 eV): $m / z=186,184\left(24,24 \%, \mathrm{M}^{+}\right), 105(100)$. ${ }^{1} \mathrm{H}-\mathrm{NMR}$ : Tab. 1. $-{ }^{13} \mathrm{C}-\mathrm{NMR}\left(\mathrm{CDCl}_{3}\right): \delta=12.81$ (d, $200 \mathrm{~Hz}$; C-1,8), 27.42 (t, $126 \mathrm{~Hz}$; C-6), 38.97 (t, $130 \mathrm{~Hz}$; C-5), 48.11 und 49.68 (jeweils d, $\approx 150 \mathrm{~Hz}$; C-2,7), 122.45 (s; C-4), 133.09 (d, $160 \mathrm{~Hz} ; \mathrm{C}-3)$.

\section{$\mathrm{C}_{8} \mathrm{H}_{9} \mathrm{Br}$ (185.1) Ber. C 51.92 H 4.90 Gef. C 51.76 H 4.93}

Tricyclo[5.1.0.0 $0^{2.8}$ ]oct-3-en (4): Unter Stickstoff legte man $1.14 \mathrm{~g}$ (49.6 mmol) Natrium in $30 \mathrm{ml}$ siedendem Ether vor und tropfte unter Rühren $2.30 \mathrm{~g}$ (12.4 mmol) 9 in $3.93 \mathrm{~g} \mathrm{(53.1}$ mmol) tert-Butylalkohol und $55 \mathrm{ml}$ Ether innerhalb von $30 \mathrm{~min} z$. Nach 48 h Rückflußkochen ließ man abkühlen, hydrolysierte vorsichtig mit Eiswasser, trennte die Phasen, extrahierte die wäßrige Phase zweimal mit je $50 \mathrm{ml}$ Ether, wusch die vereinigten organischen Phasen mit Wasser, trocknete sie über $\mathrm{Na}_{2} \mathrm{SO}_{4}$ und versetzte sie dann zwecks Entfernung von tert-Butylalkohol mit Natriumhydrid (80proz. in Paraffinöl), bis keine Gasentwicklung mehr auftrat. Der Ether wurde über eine $40-\mathrm{cm}$-Vigreuxkolonne abdestilliert. Aus dem Rückstand erhielt man bei $20^{\circ} \mathrm{C} / 0.05$ Torr $2.17 \mathrm{~g}$ eines Gemisches aus Ether und 4 als Kondensat in einer auf $-78^{\circ} \mathrm{C}$ gekühlten Vorlage. Durch Destillation über eine 8-cmVigreuxkolonne entfernte man den restlichen Ether, und $780 \mathrm{mg}(59 \%)$ des praktisch reinen 4 gingen bei $48^{\circ} \mathrm{C} / 30$ Torr als farblose Flüssigkeit über. Für die Photoelektronenspektroskopie ${ }^{13)}$ wurde eine Probe zusätzlich durch präp. Gaschromatographie gereinigt (Säule $6 \mathrm{~m}, 5 \% \mathrm{NaOH}$ und $20 \%$ Carbowax $20 \mathrm{M}$ auf Chromosorb P/NAW, $85^{\circ} \mathrm{C}$ ). - IR (Film): $1659 \mathrm{~cm}^{-1}(\mathrm{C}=\mathrm{C}) .-\mathrm{MS}(70 \mathrm{eV}): m / z=106\left(25 \%, \mathrm{M}^{+}\right), 91(81), 78(100) .-{ }^{1} \mathrm{H}-\mathrm{NMR}$ : Tab. 1. - ${ }^{13}$ C-NMR: Lit. ${ }^{16)}$.

$$
\mathrm{C}_{8} \mathrm{H}_{10} \text { (106.2) Ber. C } 90.50 \text { H } 9.50 \text { Gef. C } 90.50 \text { H } 9.78
$$

Tricyclo[5.1.0.0.8. ]octan (6): $\mathrm{Zu} 201 \mathrm{mg}(1.89 \mathrm{mmol}) 4$ in $20 \mathrm{ml}$ Ether gab man $1.13 \mathrm{~g}$ (3.79 mmol) 2,4,6-Triisopropylbenzolsulfonsäurehydrazid ${ }^{18}$ und $100 \mathrm{mg}(0.72 \mathrm{mmol}) \mathrm{K}_{2} \mathrm{CO}_{3}$, kochte 24 h unter Rühren und RückfluB, hydrolysierte, trennte die Phasen, extrahierte die wäßrige Phase zweimal mit je $10 \mathrm{ml}$ Ether, trocknete die vereinigten Etherphasen mit $\mathrm{Na}_{2} \mathrm{SO}_{4}$, destillierte das Solvens über eine $20-\mathrm{cm}$-Vigreuxkolonne ab und kondensierte die flüchtigen Komponenten des Rückstandes bei 0.01 Torr in eine auf $-78^{\circ} \mathrm{C}$ gekühlte Vorlage. Ein 'H-NMR-Spektrum zeigte die Gegenwart von 6 und 10 neben viel Ether an. Bei der präp. Gaschromatographie (Bedingungen wie bei 4) wurde 10 nicht von 6 getrennt, und es fielen $120 \mathrm{mg}$ einer farblosen Flüssigkeit an, die laut NMR-Spektrum aus 6 (52\% Ausb.)

Chem. Ber. 119 (1986) 
und $10 \mathrm{im}$ Verhältnis $8: 1$ bestand. - MS $(70 \mathrm{eV}): m / z=108\left(33 \%, \mathrm{M}^{+}\right), 93(78), 79(100)$. 'H-NMR: 6: Tab. 1; 10: Das Spektrum stimmt mit den Angaben in Lit. ${ }^{41)}$ überein. $-{ }^{13} \mathrm{C}$ NMR: 6: Lit. ${ }^{16)}$.

$$
\mathrm{C}_{8} \mathrm{H}_{12} \text { (108.2) Ber. C 88.82 H } 11.18 \text { Gef. C } 89.31 \text { H } 11.22
$$

Bicyclo[4.2.0 Jocta-3,7-dien (11): Unter Rühren tropfte man während $1 \mathrm{~h} 10.1 \mathrm{~g}$ (23.8 mmol) 7 in $200 \mathrm{ml}$ absol. Ether zur Lösung von $2.20 \mathrm{~g}(95.7 \mathrm{mmol})$ Natrium in $200 \mathrm{ml}$. กüssigem Ammoniak, die auf $-78^{\circ} \mathrm{C}$ gekühlt war. Man rührte weitere $3 \mathrm{~h}$ bei $-78^{\circ} \mathrm{C}$, setzte $\mathrm{NH}_{4} \mathrm{Cl} 2 \mathrm{u}$, bis die blaue Farbe verschwunden war, ließ das Ammoniak verdampfen, gab bis zur vollständigen Auflösung der Salze Wasser zu, trennte die Phasen, extrahierte die wäßrige Phase dreimal mit je $25 \mathrm{ml}$ Ether, trocknete die vereinigten Etherphasen mit $\mathrm{Na}_{2} \mathrm{SO}_{4}$, destillierte das Solvens über eine $20-\mathrm{cm}$-Vigrcuxkolonne ab und kondensierte die flüchtigen Bestandteile des Rückstandes bei 0.1 Torr in eine auf $-78^{\circ} \mathrm{C}$ gekühite Vorlage. Aus dem Kondensat erhielt man durch präp. Gaschromatographie (Bedingungen wie bei 4) $85 \mathrm{mg}$ einer farblosen Flüssigkeit, die zu ca. $70 \%$ aus 11 ( $2 \%$ Ausb.) bestand. Das 'H-NMRSpektrum stimmt mit den Angaben in Lit. ${ }^{27}$ überein. $-{ }^{13} \mathrm{C}-\mathrm{NMR}\left(\mathrm{CDCl}_{3}\right): \delta=26.53$ (t; C-2,5), 40.61 (d; C-1,6), 125.66 (d; C-3,4), 137.61 (d; C-7,8).

3,exo-7,syn-8-Tribrom-2-iodbicyclo[4.1.1]oct-3-en (12): In $20 \mathrm{ml}$ absol. Aceton erwärmte man $1.01 \mathrm{~g}$ ( $2.38 \mathrm{mmol}) 7$ und $447 \mathrm{mg}$ ( $2.98 \mathrm{mmol}$ ) Natriumiodid (oder $2.98 \mathrm{mmol}$ Kaliumiodid) unter Rühren $3 \mathrm{~h}$ auf $40^{\circ} \mathrm{C}$. Nach Abkühlen gab man je $50 \mathrm{ml}$ Wasser und Ether zu, trennte die Phasen, extrahierte die wäßrige Phase dreimal mit je $20 \mathrm{ml}$ Ether, wusch die vereinigten organischen Phasen zweimal mit je $20 \mathrm{ml}$ Wasser, trocknete sie über $\mathrm{Na}_{2} \mathrm{SO}_{4}$ und engte sie i.Vak. ein. Der kristallisierte Rückstand wurde aus Methanol umgelöst und erbrachte $550 \mathrm{mg}$ eines Gemisches aus 7 und 12 (44\% Ausb.) im Verhältnis 1:7.8, Schmp. $119-123^{\circ} \mathrm{C}$. - MS $(70 \mathrm{eV})$ : Im Vergleich $\mathrm{zu}$ dem von $7^{12 \mathrm{~b})}$ ist die Intensität der Liniengruppe bei $m / z=424\left(\mathrm{M}^{+}\right.$von 7$)$ stark reduziert und die Linie bei $127\left(21 \%, I^{+}\right)$neu. 'H-NMR $\left(\mathrm{CDCl}_{3}\right): \delta=2.11$ (dddd, $J_{2,5}=1.3, J_{4,5}=5.0, J_{5,5}=19.8, J_{5,6}=4.0 \mathrm{~Hz}$ ) und $2.50\left(\mathrm{dtd}, J_{2,5}=2.0, J_{4,5}=J_{5,6}=4.0 \mathrm{~Hz}\right)\left(5-\mathrm{H}_{2}\right), 3.09\left(\approx \mathrm{dq}, J_{1,6}=5.0, J_{6,8}=7.6 \mathrm{~Hz} ; 6-\mathrm{H}\right)$, 3.52 (dtd, $J_{1,2}=5.0, J_{1,7}=1.3, J_{1,8}=7.6 \mathrm{~Hz} ; 1-\mathrm{H}$ ), 4.28 (br. s; 7-H), 5.07 (br. d; $2-\mathrm{H}$ ), 5.11 (t; 8-H), 6.15 (dd; 4-H). $-{ }^{13} \mathrm{C}-\mathrm{NMR}\left(\mathrm{CDCl}_{3}\right): \delta=33.30$ (t; C-5), 37.91 (d; C-2), 43.65 und 47.80 (jeweils d; C-7,8), 49.06 (d; C-6), 59.60 (d; C-1), 124.02 (s; C-3), 130.42 (d; C-4).

\section{$\mathrm{C}_{8} \mathrm{H}_{8} \mathrm{Br}_{3} \mathrm{I}$ (470.8) Ber. C 20.41 H 1.71 Gef. C 21.74 H 1.76}

(Abweichung zum Teil bedingt durch den Anteil von 7)

4,exo-7.syn-8-Tribrombicyclo[4.1.1]oct-4-en-endo-3-yl-nitrat (13): Man kochte $5.04 \mathrm{~g}$ (11.9 mmol) 7 und $2.10 \mathrm{~g}(12.4 \mathrm{mmol})$ Silbernitrat in $20 \mathrm{ml}$ absol. Aceton $4 \mathrm{~h}$ unter Rückfluß, ließ abkühlen, versetzte dann mit $30 \mathrm{ml}$ Ether, filtrierte einen Niederschlag $(\mathrm{AgBr}) \mathrm{ab}$, gab zum Filtrat $40 \mathrm{ml}$ Wasser, trennte die Phasen, extrahierte die wäBrige Phase dreimal mit je $30 \mathrm{ml}$ Ether, wusch die vereinigten organischen Phasen zweimal mit je $25 \mathrm{ml}$ Wasser und trocknete sie über $\mathrm{Na}_{2} \mathrm{SO}_{4}$. Das nach Abdampfen des Ethers i.Vak. zurückbleibende zähe, rote $\mathrm{Ol}_{1}$ wurde in heißem Methanol gelöst; beim Abkühlen schieden sich $2.50 \mathrm{~g}(52 \%) 13$ als farblose Kristalle mit Schmp. $74-75^{\circ} \mathrm{C}$ ab. Man engte die Mutterlauge i. Vak. ein, nahm den Rückstand mit Cyclohexan auf, filtrierte über Aluminiumoxid (Akt.-Stufe III), engte erneut ein und erhielt durch Behandlung des Rückstands mit $3 \mathrm{ml}$ Methanol weitere $1.10 \mathrm{~g} \mathrm{(22 \% )}$ 13. - IR (KBr): $1635 \mathrm{~cm}^{-1}(\mathrm{C}=\mathrm{C})$. - MS (70 eV): $m / z=407,405\left(0.2,0.2 \%, \mathrm{M}^{+}\right), 91$ (100). - 'H-NMR $\left(\mathrm{CDCl}_{3}\right): \delta=2.46$ (ddd, $J_{1,2 e \times 0}=7.5, J_{2,2}=16.5, J_{2 e \times 0.3}=1.9 \mathrm{~Hz}$; $2-\mathrm{H}_{\text {exo }}$ ), 2.62 (ddd, $J_{1,2 e n d o}=1.5, J_{2 e n d 0.3}=6.0 \mathrm{~Hz}, 2-\mathrm{H}_{\text {endo }}$ ), 3.12 (br. dt, $J_{1,6}=6.0, J_{1,8}=$ $7.3 \mathrm{~Hz} ; 1-\mathrm{H}$ ), 3.43 (dddd, $J_{5,6}=9.8, J_{6,7}=0.7, J_{6,8}=7.3 \mathrm{~Hz} ; 6-\mathrm{H}$ ), 4.52 (br. s; 7-H), 5.48 (br. t; 8-H), 5.97 (dd; 3-H), 6.57 (d; 5-H). $-{ }^{13} \mathrm{C}-\mathrm{NMR}\left(\mathrm{CDCl}_{3}\right): \delta=31.19$ (t; C-2), 43.46 
und 47.90 (jeweils d; C-7,8), 49.63 und 52.81 (jeweils d; C-1,6), 85.08 (d; C-3), 119.04 (s; C-4), 138.38 (d; C-5).

$\mathrm{C}_{8} \mathrm{H}_{8} \mathrm{Br}_{3} \mathrm{NO}_{3}$ (405.9) Ber. C 23.67 H $1.99 \mathrm{~N} 3.45$ Gef. C 24.22 H 1.89 N 3.67

3,exo-7,syn-8-Tribrombicyclo[4.1.1]oct-3-en (14): Zu $3.50 \mathrm{~g}$ (8.62 mmol) $13 \mathrm{in} 50 \mathrm{ml}$ absol. Ether gab man bei $20^{\circ} \mathrm{C}$ unter Rühren innerhalb von $5 \mathrm{~min} 655 \mathrm{mg}(17.2 \mathrm{mmol})$ Lithiumaluminiumhydrid, rührte dann $30 \mathrm{~min}$ bei $20^{\circ} \mathrm{C}$, kochte $1 \mathrm{~h}$ unter RückfluB, hydrolysierte nach dem Abkühlen vorsichtig, trennte die Phasen, extrahierte die wäßrige Phase dreimal mit je $25 \mathrm{ml}$ Ether, trocknete die vereinigten Etherphasen über $\mathrm{Na}_{2} \mathrm{SO}_{4}$ und engte i.Vak. ein. Aus dem Rückstand destillierten bei $40-50^{\circ} \mathrm{C}(\mathrm{Bad}) / 0.001$ Torr $2.47 \mathrm{~g}(83 \%) 14$ als farbloses Öl. - IR (Film): $1659 \mathrm{~cm}^{-1}(C=C)$ - MS (70 eV): $m / z=348,346,344,342$ (4, $\left.11,12,4 \%, \mathrm{M}^{+}\right), 104(100)$. $-{ }^{1} \mathrm{H}-\mathrm{NMR}\left(\mathrm{CDCl}_{3}\right): \delta=2.33\left(\approx \mathrm{dtt}, J_{\text {cis-2.,5 }} \approx J_{\text {trans-2.5 }} \approx 2.5\right.$, $\left.J_{4,5} \approx J_{5,6} \approx 3.5, J_{5,5}=19.0 \mathrm{~Hz}\right)$ und $2.57\left(\approx \mathrm{dtt}, J_{\text {cis-2,5 }} \approx J_{\text {trans-2,5 }} \approx 2.3, J_{4,5} \approx J_{5,6} \approx\right.$ $4.5 \mathrm{~Hz})\left(5-\mathrm{H}_{2}\right), 2.74$ (ddq, $\left.J_{1,2}=4.5, J_{2,2}=20.0, J_{2,4}=2.4 \mathrm{~Hz}\right)$ und $2.99(\mathrm{dm})\left(2-\mathrm{H}_{2}\right), 3.01$ $(\mathrm{m} ; 1-\mathrm{H}), 3.09$ ( $\left.\approx \mathrm{dqt}, J_{1,6}=4.5, J_{4,6}=J_{6,7}=1.0, J_{6,8}=7.8 \mathrm{~Hz} ; 6-\mathrm{H}\right), 4.29\left(\mathrm{q}, J_{1,7}=J_{7,8}=\right.$ $1.0 \mathrm{~Hz} ; 7-\mathrm{H}), 5.34$ (br. t, $\left.J_{1,8}=7.8 \mathrm{~Hz} ; 8-\mathrm{H}\right), 6.07(\mathrm{~m} ; 4-\mathrm{H}) .-{ }^{13} \mathrm{C}-\mathrm{NMR}\left(\mathrm{CDCl}_{3}\right): \delta=31.92$ (t; C-5), 40.47 (t; C-2), 44.36 und 48.12 (jeweils d; C-7,8), 50.08 und 50.53 (jeweils $d, C-1,6$ ), 119.81 (s; C-3), 128.36 (d; C-4).

\section{$\mathrm{C}_{8} \mathrm{H}_{9} \mathrm{Br}_{3}$ (344.9) Ber. C 27.86 H 2.63 Gef. C 28.01 H 2.49}

4-Bromtricyclo[5.1.0.0 2.8 ]oct-4-en (15): Unter Stickstoff tropite man zur gerührten Lösung von $1.91 \mathrm{~g}$ (5.54 mmol) 14 in $10 \mathrm{ml}$ absol. Ether, die auf $-70^{\circ} \mathrm{C}$ gekühlt war, innerhalb von $10 \mathrm{~min} 6.09 \mathrm{mmol} \mathrm{n}$-Butyllithium (1.5 $\mathrm{N}$ in Hexan). Bei fortgesetztem Rühren lieB man während $1 \mathrm{~h}$ auf $0^{\circ} \mathrm{C}$ erwärmen, hydrolysierte, trennte die Phasen, extrahierte die wäßrige Phase zweimal mit je $20 \mathrm{ml}$ Ether, trocknete die vereinigten Etherphasen über $\mathrm{Na}_{2} \mathrm{SO}_{4}$ und engte i. Vak. ein. Aus dem Rückstand destillierten bei $60-65^{\circ} \mathrm{C}(\mathrm{Bad}) / 1$ Torr $595 \mathrm{mg}(58 \%)$ 15 als farblose Flüssigkeit. - MS (70 eV): $m / z=186,184\left(5,5 \%, \mathrm{M}^{+}\right), 105(100) .-{ }^{\prime} \mathrm{H}$ NMR: Tab. 1. $-{ }^{13} \mathrm{C}-\mathrm{NMR}\left(\mathrm{CDCl}_{3}\right): \delta=12.25$ (d, $\left.195 \mathrm{~Hz} ; \mathrm{C}-1,8\right), 33.12$ (t, $\left.126 \mathrm{~Hz} ; \mathrm{C}-6\right)$, 44.99 (t, $128 \mathrm{~Hz}$; C-3), 50.72 und 50.77 (jeweils d, $148 \mathrm{~Hz}$; C-2,7), 121.38 (s; C-4), 130.12 (d, $161 \mathrm{~Hz} ; \mathrm{C}-5)$.

\section{$\mathrm{C}_{8} \mathrm{H}_{9} \mathrm{Br}(185.1)$ Ber. C 51.92 H 4.90 Gef. C 51.38 H 5.06}

Tricyclo[ 5.1.0.0.8.8 Joct-4-en (5): Man tropfte eine Lösung von $485 \mathrm{mg}$ (2.62 mmol) 15 und $777 \mathrm{mg}(10.5 \mathrm{mmol})$ tert-Butylalkohol in $20 \mathrm{ml}$ absol. Ether innerhalb von 5 min unter Rühren zu $120 \mathrm{mg}$ ( $5.20 \mathrm{mmol})$ Natrium in $10 \mathrm{ml}$ siedendem Ether, erhitzte unter weiterem Rühren 2 d unter RückfluB, hydrolysierte nach Abkühlen, trennte die Phasen, extrahierte die wäBrige Phase zweimal mit je $5 \mathrm{ml}$ Ether, wusch die vereinigten organischen Phasen zweimal mit je $20 \mathrm{ml}$ Wasser, trocknete sie über $\mathrm{Na}_{2} \mathrm{SO}_{4}$, destillierte den Ether über eine 20-cm-Vigreuxkolonne ab und kondensierte flüchtige Komponenten des Rückstandes bei $20^{\circ} \mathrm{C} / 0.05$ Torr in eine auf $-78^{\circ} \mathrm{C}$ gekühlte Vorlage. Mit Hilfe der präp. Gaschromatographie (Bedingungen wie bei 4$)$ wurden $85 \mathrm{mg}(31 \%)$ reines 5 als farblose Flüssigkeit isoliert. MS $(70 \mathrm{eV}): m / z=106\left(25 \%, \mathrm{M}^{+}\right), 91(100), 78(83)$ - ${ }^{1} \mathrm{H}-\mathrm{NMR}$ : Tab. 1. - ${ }^{13} \mathrm{C}-\mathrm{NMR}$ : Lit. ${ }^{16)}$.

\section{$\mathrm{C}_{8} \mathrm{H}_{10}$ Ber. 106.0783 Gef. 106.0787}

Tricyclo[ 5.1.0.0.8.8 Jocta-3.5-dien (1): Unter Stickstoff tropfte man $2.16 \mathrm{mmol}$ tert-Butyllithium (1.3 N in Pentan) innerhalb von $10 \mathrm{~min} 2 \mathrm{zu} 198 \mathrm{mg}$ (1.08 mmol) 4-Bromoctavalen $(16)^{12 b)}$ in $3 \mathrm{ml}$ absol. Ether, der bei $-78^{\circ} \mathrm{C}$ gerührt wurde. Man setzte das Rühren $30 \mathrm{~min}$ fort und gab bei $-78^{\circ} \mathrm{C} 5 \mathrm{ml}$ Wasser zu. Nach dem Auftauen trennte man die Phasen, extrahierte die wäßrige Phase zweimal mit je $5 \mathrm{ml}$ Ether, trocknete die vereinigten organischen Phasen über $\mathrm{Na}_{2} \mathrm{SO}_{4}$ und verdampfte das Solvens bei $0^{\circ} \mathrm{C} / 20$ Torr. Bei $20^{\circ} \mathrm{C} / 0.02$

Chem. Ber. 119 (1986) 
Torr verdampfte man die flüchtigen Anteile des Rückstandes und kondensierte sie in einer auf $-180^{\circ} \mathrm{C}$ gekühlten Vorlage. Man erhielt $65 \mathrm{mg}$ einer farblosen Flüssigkeit, die im wesentlichen aus $1(57 \%)$ bestand. Das 'H-NMR-Spektrum enthielt einige Fremdsignale bei $\delta=0.7-1.1$, deren Gesamtintegral so groß wie jenes eines Zwei-Protonen-Signals von 1 war. Nach einer Woche bei $-20^{\circ} \mathrm{C}$ lag das Verhältnis 1: Cyclooctatetraen bei 64:1. - ${ }^{1} \mathrm{H}$ NMR: Tab. 1. - ${ }^{13}$ C-NMR: Lit. ${ }^{12,16}$.

\section{B. Thermolysen der Kohlenwasserstofie 4-6}

Die Lösung von 10-40 mg des betreffenden Kohlenwasserstoffs in ca. $0.4 \mathrm{ml} \mathrm{C}_{6} \mathrm{D}_{6}$ wurde mit $200 \mathrm{mg}$ Lithiumaluminiumhydrid versetzt und $1 \mathrm{~h}$ unter Stickstoff gerührt, was der Entfernung von Säurespuren dienen sollte. Bei $10^{-3}$ Torr dampfte man dann $C_{6} D_{6}$ und Kohlenwasserstoff $\mathrm{ab}$, kondensierte sie in ein auf $-180^{\circ} \mathrm{C}$ gekühltes NMR-Röhrchen, schmolz dieses nach mehrmaligem Entgasen i.Vak. ab und brachte es zur Thermolyse in einen Trockenschrank mit der im jeweiligen Fall angegebenen Temperatur. Zur Kontrolle des Reaktionsverlaufs wurde von Zeit zu Zeit ein 'H-NMR-Spektrum aufgenommen, wobei das $\mathrm{C}_{6} \mathrm{D}_{5} \mathrm{H}$-Signal als interner Standard zur Konzentrationsbestimmung Verwendung fand.

Thermolyse von Tricyclo[5.1.0.0.8 Joct-3-en (4): Bei $100^{\circ} \mathrm{C}$ bildete sich mit einer Halbwertszeit von ca. $40 \mathrm{~min}$ und nahezu quantitativer Ausb. Dihydrosemibullvalen (17). Das ${ }^{1} \mathrm{H}-\mathrm{NMR}$-Spektrum stimmte mit Literaturangaben ${ }^{27}$ überein. Durch Entkopplungsexperimente im 400-MHz- ${ }^{1} \mathrm{H}-\mathrm{NMR}$-Spektrum konnten alle Parameter ermittelt und zugeordnet werden; siehe Tab. 2. Diese Daten stehen mit jenen substituierter DihydrosemibullvalenDerivate ${ }^{42)}$ in Einklang. - ${ }^{13} \mathrm{C}-\mathrm{NMR}\left(\mathrm{C}_{6} \mathrm{D}_{6}\right): \delta=22.51$ und 41.09 (jeweils $\mathrm{t}, 129 \mathrm{~Hz}$; C-6,7); 28.84 (d, $163 \mathrm{~Hz}$ ), 33.03 (d, $166 \mathrm{~Hz}$ ), 36.58 (d, $173 \mathrm{~Hz}$ (C-1,2,8); 49.47 (d, $140 \mathrm{~Hz}$; C-5); 127.00 und 133.37 (jeweils d, $163 \mathrm{~Hz}$; C-3,4).

Tab. 2. ${ }^{1} \mathrm{H}-\mathrm{NMR}$-Chemische Verschiebungen ( $\delta$-Werte, Diagonalelemente, kursiv) und Kopplungskonstanten (Absolutwerte in $\mathrm{Hz}$, Nichtdiagonalelemente) von Dihydrosemibullvalen (17) in $\mathrm{C}_{6} \mathrm{D}_{6}$. Leere Felder bedeuten $J<0.5 \mathrm{~Hz}$

\begin{tabular}{|c|c|c|c|c|c|c|c|c|c|c|}
\hline & $1-\mathrm{H}$ & $2-H$ & $3-\mathrm{H}$ & $4-\mathrm{H}$ & $5-\mathrm{H}$ & 6- $\mathrm{H}_{\text {endo }}$ & $6-\mathrm{H}_{\text {exo }}$ & 7- $\mathrm{H}_{\text {endo }}$ & $7 \cdot \mathrm{H}_{\text {exo }}$ & 8-H \\
\hline $\begin{array}{l}1-\mathrm{H} \\
2-\mathrm{H} \\
3-\mathrm{H} \\
4-\mathrm{H} \\
5-\mathrm{H} \\
6-\mathrm{H}_{\text {endo }} \\
6-\mathrm{H}_{\text {exo }} \\
7-\mathrm{H}_{\text {endo }} \\
7-\mathrm{H}_{\text {exo }} \\
8-\mathrm{H}\end{array}$ & 2.33 & $\begin{array}{l}6.8 \\
1.70\end{array}$ & $\begin{array}{l}2.2 \\
5.51\end{array}$ & $\begin{array}{c}\approx 0.5 \\
\approx 0.5 \\
5.3 \\
5.33\end{array}$ & $\begin{array}{l}5.8 \\
0.7 \\
\\
2.2 \\
2.95\end{array}$ & $\begin{array}{c}\approx 0.5 \\
1.38\end{array}$ & $\begin{array}{c}5.2 \\
11.1 \\
1.82\end{array}$ & $\begin{array}{c}6.0 \\
11.1 \\
1.59\end{array}$ & $\begin{array}{c}1.3 \\
\approx 0.5 \\
8.8 \\
13.1 \\
1.72\end{array}$ & $\begin{array}{r}7.0 \\
7.0 \\
0.7 \\
\approx 0.5\end{array}$ \\
\hline
\end{tabular}

Thermolyse von Tricycio[ 5.1.0.0.8.8 ]oct-4-en (5): Bei $200^{\circ} \mathrm{C}$ zerfiel 5 mit einer Halbwertszeit von ca. $25 \mathrm{~h}$ zu einem komplexen Gemisch, in dem als eine mengenmäßig wichtige Komponente 11 durch Vergleich der NMR-Spektren mit denen der authentischen Substanz identifiziert wurde. Die weiteren Bestandteile verursachen im 'H-NMR-Spektrum $\left(C_{6} D_{6}\right)$ Absorptionen bei $\delta=0.3-1.6,2.0-3.0,4.7-6.2$, wobei sehr breite Banden im zweiten und dritten Bereich auf Polymere hindeuten. Die Multiplizität der Signale im ersten Bereich läBt auf ein cis-disubstituiertes Cyclopropan schließen. Im ${ }^{13} \mathrm{C}$-NMR-Spektrum finden sich über

Chem. Ber. 119 (1986) 
die Linien von 11 hinaus die acht wichtigsten Signale bei $\delta=28.4,29.9,33.3,35.1,35.6$, 36.5, 39.2, 51.9.

Thermolyse von Tricyclo[5.1.0.0.8 ]octan (6): Bei $160^{\circ} \mathrm{C}$ zerfiel 6 mit einer Halbwertszeit von ca. $8 \mathrm{~h}$ unter Bildung eines 5.5:1-Gemisches aus 3-Methylencyclohepten (18) und 1,3Cyclooctadien (19) mit nahezu quantitativer Ausbeute. Weitere im 400-MHz-' $\mathrm{H}-\mathrm{NMR}$ Spektrum erkennbare Produkte waren mengenmäßig unbedeutend. Der mit 6 eingebrachte Anteil an Bicyclo[5.1.0]oct-2-en (10) schien sich nicht verändert zu haben. Das 'H-NMRSpektrum von $18^{28)}$ und das ${ }^{13} \mathrm{C}$-NMR-Spektrum von $19^{291}$ stimmen mit Literaturangaben überein. - ${ }^{13} \mathrm{C}-\mathrm{NMR}\left(\mathrm{C}_{6} \mathrm{D}_{6}\right)$ von 18: $\delta=27.53,28.18,28.78$ und 36.04 (jeweils $\left.t ; C-4,5,6,7\right)$; $114.89\left(\mathrm{t} ;=\mathrm{CH}_{2}\right) ; 131.87$ und 133.33 (jeweils $\left.\mathrm{d} ; \mathrm{C}-1,2\right) ; 148.20(\mathrm{~s} ; \mathrm{C}-3)$.

\section{CAS-Registry-Nummern}

1: 35438-35-8 / 4: 102575-26-8 / 5: 102575-25-7 / 6: 36328-29-7 / 7: 103148-56-7 /8: 103148 57-8 / 9: 103148-58-9 / 10: 56797-66-1 / 11: 103148-59-0 / 12: 103148-60-3 / 13: 103148-61. 4 / 14: 103148-62-5 / 15: 103148-63-6 / 16: 103148-64-7 / 17: 3725-33-5 / 18: 34564-56-2 / 19: $1700-10-3$

1) Aus der Dissertation C. Herzog, Univ. Würzburg 1986.

2) $P$. Bischof, R. Gleiter und E. Müller, Tetrahedron 32, 2769 (1976).

3) A. Schloßer, F. Philipp, W. Mickler, G. Szeimies und H.-D. Martin, Chem. Ber. 113, 1053 (1980).

4) M. N. Paddon-Row, K. N. Houk, P. Dowd, P. Garner und R. Schappert, Tetrahedron Lett. 22, 4799 (1981).

s) P. G. Gassman, M. L. Greenlee, D. A. Dixon, S. Richtsmeier und J. Z. Gougoutas, J. Am. Chem. Soc. 105, 5865 (1983).

6) K. W. Cox, M. D. Harmony, G. Nelson und K. B. Wiberg, J. Chem. Phys. 50, 1976 (1969).

${ }^{7} H$. Irngartinger, A. Goldmann, R. Jahn, M. Nixdorf, H. Rodewald, G. Maier, K.-D. Malsch und R. Emrich, Angew. Chem. 96, 967 (1984); Angew. Chem., Int. Ed. Engl. 23, 993 (1984).

8) 8a) C. S. Gibbons und J. Trotter, J. Chem. Soc. A 1967, 2027. - 8b) H. Irngartinger und K. L. Lukas, Angew. Chem. 91, 750 (1979); Angew. Chem., Int. Ed. Engl. 18, 694 (1979). 8c) P. Dowd, P. Garner, R. Schappert, H. Irngartinger und A. Goldmann, J. Org. Chem. 47, 4240 (1982).

9) R. D. Suenram und M. D. Harmony, J. Am. Chem. Soc. 95, 4506 (1973); R. R. Karl jr. und S. H. Bauer, J. Mol. Struct. 25, 1 (1975).

10) M. Christl, B. Mattauch. H. Imgartinger und A. Goldmann, Chem. Ber. 119, 950 (1986).

11) C. Kabuto, T. Tatsuoka, I. Murata und Y. Kitahara, Angew. Chem. 86, 738 (1974); Angew. Chem., Int. Ed. Engl. 13, 669 (1974).

12) 12a) $M$. Christl und R. Lang. J. Am. Chem. Soc. 104, 4494 (1982). - 12b) M. Christl, R. Lang und C. Herzog, Tetrahedron 42, 1585 (1986).

13) $R$. Gleiter, P. Bischof und M. Christl, J. Org. Chem. 51, 2895 (1986)

14) $M$. Christl, R. Lang, W. Reimann und H. Ingartinger, Chem. Ber. 117, 959 (1984).

15) T. Matsui und M. Nakayama, Bull Chem. Soc. Jpn. 56, 3531 (1983).

16) $M$. Christl und $C$. Herzog, Chem. Ber. 119, 3067 (1986).

17) 17a) $M$. Christl und G. Brüntrup. Chem. Ber. 107, 3908 (1974). - 17b) M. Christl, P. Kemmer und B. Mattauch, Chem. Ber. 119, 960 (1986).

18) N. J. Cusack, C. B. Reese und B. Roozpeikar, J. Chem. Soc., Chem. Commun. 1972, 1132; N. J. Cusack, C. B. Reese, A. C. Risius und B. Roozpeikar, Tetrahedron 32, 2157 (1976).

19) 19a) W. R. Moore, H. R. Ward und R. F. Merritt, J. Am. Chem. Soc. 83, 2019 (1961); M. $C h r i s t l$ und $M$. Lechner, Chem. Ber. 115, 1 (1982), und dort zitierte Literatur. - ${ }^{196} M$. Christl, U. Heinemann und W. Kristof, J. Am. Chem. Soc. 97,2299 (1975).

${ }^{20)} F$. Wille, $K$. Dirr und $H$. Kerber, Liebigs Ann. Chem. 591, 177 (1955).

${ }^{21)}$ L. Fishbein und J. A. Gallaghan, J. Am. Chem. Soc. 78, 1218 (1956).

${ }^{22)}$ L. M. Soffer, E. W. Parrotta und J. Di Domenico, J. Am. Chem. Soc. 74, 5301 (1952).

${ }^{23)} G$. W. H. Cheeseman, J. Chem. Soc. 1957, 115.

24) M. Christl, C. Herzog, D. Brückner und R. Lang. Chem. Ber. 119, 141 (1986).

Chem. Ber. 119 (1986) 
25) R. M. Magid, Tetrahedron 36, 1901 (1980).

26) $\mathrm{H}$. Günther, NMR-Spektroskopie, 2. Aufl., Thieme, Stuttgart 1983.

27) W. R. Roth und B. Peltzer, Liebigs Ann. Chem. 685, 56 (1965).

28) G. S. Groenewold und M. L. Gross, J. Am. Chem. Soc. 106, 6569 (1984)

29) H. O. Kalinowski, S. Berger und S. Braun, ${ }^{13}$ C-NMR-Spektroskopie, Thieme, Stuttgart 1984.

30) M. J. S. Dewar und S. Kirschner, J. Am. Chem. Soc. 97, 2931 (1975).

${ }^{31)} R$. Stangl, Dissertation, Univ. Würzburg 1984.

32) W. R. Roth und B. Peltzer, Angew. Chem. 76, 378 (1964); Angew. Chem., Int. Ed. Engl. 3, 440 (1964).

33) G. L. Closs und P. E. Pfeffer, J. Am. Chem. Soc. 90, 2452 (1968); D. P: G. Hamon, Aust. J. Chem. 27, 153 (1974); 28, 2641 (1975).

34) 342) $K$. B. Wiberg und $G$. Szeimies, Tetrahedron Lett. 1968, 1235. - ${ }^{346)}$ Abweichend von Lit. ${ }^{196)}$ beträgt unter sorgfăligem Ausschluß von Säurespuren die Ausbeute für die Umlagerung $24 \rightarrow 26$ in Lösung mehr als $90 \%: M$. Christl, Habilitationsschrift, Univ. Würzburg 1977.

35) R. Criegee, D. Seebach, R. E. Winter, B. Börretzen und H.-A. Brune, Chem. Ber. 98, 2339 (1965).

36 K.-D. Baumgart, H. Harnisch, U. Szeimies-Seebach und G. Szeimies, Chem. Ber. 118, 2883 (1985).

${ }^{37}$ L. A. Paquette und G. Zon, J. Am. Chem. Soc. 96, 203 (1974); G. Zon und L. A. Paquette, ebenda 96, 215 (1974), und dort zitierte Literatur.

38) M. Christl, Angew. Chem. 93, 515 (1981); Angew. Chem., Int. Ed. Engl. 20, 529 (1981), und dort zitierte Literatur.

39) H. E. Zimmerman und L. R. Sousa, J. Am. Chem. Soc. 94, 834 (1972).

40) E. Vogel, H. Kiefer und W. R. Roth, Angew. Chem. 76, 432 (1964); Angew. Chem., Int. Ed. Engl. 3, 442 (1964).

41) E. Vedejs, M. F. Salomon und P. D. Weeks, J. Am. Chem. Soc. 95,6770 (1973).

${ }^{42)} M$. Christl, E. Brunn und F. Lanzendörfer, J. Am. Chem. Soc. 106, 373 (1984); E. M. Osselton und J. Cornelisse, Tetrahedron Lett. 26, 527 (1985).

$[88 / 86]$ 